\title{
Fundamental Limits on the Uplink Performance of the Dynamic- Ordered Successive Interference Cancellation Receiver
}

This paper was downloaded from TechRxiv (https://www.techrxiv.org).

\section{LICENSE}

CC BY 4.0

SUBMISSION DATE / POSTED DATE

$13-10-2021 / 18-10-2021$

\section{CITATION}

Lusvarghi, Luca; Merani, Maria Luisa (2021): Fundamental Limits on the Uplink Performance of the DynamicOrdered Successive Interference Cancellation Receiver. TechRxiv. Preprint.

https://doi.org/10.36227/techrxiv.16803496.v1

DOI

10.36227/techrxiv.16803496.v1 


\title{
Fundamental Limits on the Uplink Performance of the Dynamic-Ordered Successive Interference Cancellation Receiver \\ Luca Lusvarghi, Student Member, IEEE, and Maria Luisa Merani, Senior Member, IEEE
}

\begin{abstract}
This work puts forth a novel analytical approach to evaluate the performance that power-domain Non-Orthogonal Multiple Access (NOMA) achieves on the uplink of a single cell. A dynamic-ordered Successive Interference Cancellation (SIC) receiver is considered, and both the case of Rayleigh and lognormal-shadowed Rayleigh fading are examined. System performance is assessed analytically, deriving either exact or approximated closed-form expressions, whose correctness and excellent accuracy are validated through Monte Carlo simulations. The analysis discloses the effects on performance of an arbitrary number $n$ of simultaneously transmitting users, therefore unveiling where the insourmountable limits of the dynamic-ordered SIC receiver lie. Moreover, the proposed methodology allows to quantify the impact of lognormal shadowing on NOMA efficacy.
\end{abstract}

\section{Index Terms}

NOMA, uplink NOMA, outage probability, order statistics, dynamic-order SIC

\section{INTRODUCTION}

Scanning the 5G and beyond horizon, wireless connectivity appears as one of the key enabling technologies for future Industrial Internet of Things (IoT). The design of such connectivity paradigm is challenged by the requirement to support a number of users that is increasing at an

The authors are with the Department of Engineering "Enzo Ferrari”, University of Modena and Reggio Emilia, Modena 41125, Italy (e-mail: luca.lusvarghi5@unimore.it; marialuisa.merani@unimore.it)

Manuscript received August 7, 2021; revised XXX, 2021. 
extraordinary pace, with every user exhibiting remarkable bandwidth requirements [1]. There is no doubt that the effectiveness of the techniques devised to share radio resources, packing more and more users on the same radio spectrum, are pushed to the limit.

Over the last years, a large debate has spurred around NOMA, breeding an abundant body of scientific literature; the aim of the research is to discover among the different flavors of this approach - be it power-domain, code or interleaving based - the pitlock to accommodate the very many communications occurring in dense environments. The NOMA firestorm recently culminated with its exclusion from the $5 \mathrm{G}$ work-items, its potential adoption being postponed to $6 \mathrm{G}$ networks. The decision had partly to be ascribed to the questioned advantage of NOMA schemes over the canonical OMA strategy, when the latter is combined with massive Multiple Input Multiple Output (MIMO) [2], a technology characterized by a number of antennas at the base station much larger than the number of simultaneously active users, that allows to spatially multiplex more communications over the same radio resources.

The risk that every NOMA bibliography incurs is to forget or inadvertently miss some relevant works among the multitude of published papers: on purpose, a few surveys are mentioned in this introduction, adhering to a temporal progression, from [3], to [4], [5] and more recently [6].

Confining the attention to power-domain NOMA, its behavior on the uplink of cellular systems has been assessed in numerous studies; we deem [7] and [8] especially meaningful contributions. Moreover, it is worth recalling the contribution in [9], where NOMA transmission paired with a novel power control scheme was studied, and the performance was analyzed in terms of outage probability and achievable data rate. Stemming from [9], [10] focused on the optimization of the power allocation strategy. The authors of [11] and [12] analyzed the outage probability of a NOMA system, examining the adoption of a dynamic-ordered SIC receiver, that is, of a receiver whose decoding order is adaptively varied on the basis of the instantaneous received powers of the simultaneous users. They obtained closed-form expressions for the case of three users, without however providing a systematic analysis. All previous investigations were performed in the exclusive presence of Rayleigh fading, an assumption that greatly simplifies the study when the number of simultaneously transmitted signals is small. The work in [13] introduced the analysis of generalized fading channels, encompassing statistics such as Rayleigh, Rice, Nakagami. Yet, the examined SIC receiver decoded users on the basis of the averaged received powers; even when this simpler decoding scheme was investigated, the authors recognized the complex nature of the analysis, and considered the circumstance of two users for mathematical 
tractability. In [14], the channel modeling was the generalized $\alpha-\mu$ fading and the user decoding order was based on the average received powers; here too, the analysis was limited to the circumstance of two or at the most three users.

In the current work, a power-domain NOMA-based cell where the base station and the users are equipped with a single antenna is considered; the choice is to mainly condense the design complexity at the base station and to examine uplink communications. The adoption of a dynamic-ordered SIC receiver is assumed. Hence, the receiver attempts to decode the signal coming from the user with the highest instantaneous received power first; if successful, it removes such signal and then proceeds to decode the second strongest, iterating the removal process at every successful decoding step. The assumption of only two superimposed signals usually found in literature is removed, to disclose the ultimate achievable performance of the receiver. Furthermore, the analysis in the presence of Rayleigh fading is paired by the investigation of the combined effects of fading and lognormal shadowing. To characterize system behavior, the outage probabilities $P_{\text {out }}^{(j)}, j=1,2, \ldots, n$, are determined, the generic $P_{\text {out }}^{(j)}$ being defined as the probability that the receiver fails to decode the $j$-th strongest signal and therefore cannot recover the remaining $n-j$ weaker signals.

This work offers several novel contributions:

- a general method to analytically evaluate the outage probabilities is provided, based on the unique properties of the joint probability density function of the ordered received powers, as the latter are dependent, non identically distributed random variables. The approach can be profitably employed for any number $n$ of simultaneously received signals;

- when Rayleigh fading is considered, the exact analytical expression of $P_{\text {out }}^{(1)}$, the probability that the decoding process does not begin and the SIC receiver fails to recover any of the simultaneous signals, is provided for an arbitrary value of $n$. An accurate, closed-form approximation of $P_{\text {out }}^{(j)}, j \geq 2$, is also put forth;

- when the signals are affected by Rayleigh-lognormal shadowed fading, an approximation of the outage probability $P_{\text {out }}^{(j)}, j \geq 1$, is offered, demonstrating that it achieves an excellent accuracy, again for an arbitrary $n$;

- the proposed approximations show that, $j$ being fixed, $P_{\text {out }}^{(j)}, j \geq 2$, is a function of the probabilities $P_{\text {out }}^{(1)}$ in the presence of $n, n-1, \ldots, n-j+1$ simultaneous signals.

Overall, the analysis discloses the limits that the dynamic-ordered SIC receiver faces for an increasing number of superimposed signals. Furthermore, it reveals that lognormal shadowing is 
responsible for a non-negligible performance worsening, with respect to the circumstance where Rayleigh fading only is considered. The deterioration is quantified for different values of $\sigma_{L}$, the standard deviation of the slow, lognormal fading in $\mathrm{dB}$.

The remainder of the paper is organized as follows. Section II introduces the system model, it illustrates the analysis and it puts forth the approximations to the outage probabilities experienced on the uplink. Section III specializes the study to the cases of Rayleigh and Rayleigh-lognormal shadowed fading. Section IV provides several numerical results that validate the approach and Section V draws the conclusions.

\section{Performance Analysis}

\section{A. System Model and Performance Metric Evaluation}

The current work focuses on the uplink communications in a cellular system. Power-domain NOMA is considered and the reference scenario features $n$ User Equipments (UEs) that transmit to a central-located base station on the same radio spectrum. Let $p_{t, i}$ denote the transmission power of the $i$-th UE and $h_{i}$ the envelope of the channel between such UE and the base station. Let

$$
X_{i}=p_{t, i}\left|h_{i}\right|^{2} \quad i=1,2, \ldots, n
$$

denote the instantaneously received power at the base station from the $i$-th UE. Further assume that every UE experiences independent channel conditions while transmitting to the base station; it follows that $h_{i}$ and $h_{j}$ are independent random variables, $\forall i$ and $j, i \neq j$, and so are $X_{i}$ and $X_{j}$. Moreover, assume that the signal recovery is performed using a dynamic-ordered SIC receiver. This choice implies that: (i) the instantaneously received signal powers from the UEs are first sorted in descending order at the base station; (ii) the receiver attempts to decode the signals in accordance to the same sequence.

Indicate by $\mathcal{S}_{\mathcal{N}}$ the $n$ ! permutations of the set $N=\{1,2, \ldots, n\}$ that identifies the $n$ UEs and by $R=\left\{r_{1}, r_{2}, \ldots, r_{n}\right\}, R \in \mathcal{S}_{\mathcal{N}}$, the permutation that corresponds to the descending order of the instantaneously received powers. It follows that

$$
p_{t, r_{1}}\left|h_{r_{1}}\right|^{2} \geq p_{t, r_{2}}\left|h_{r_{2}}\right|^{2} \geq \ldots \geq p_{t, r_{n}}\left|h_{r_{n}}\right|^{2} .
$$

Next, introduce the random variables

$$
X_{(i)}=p_{t, r_{i}}\left|h_{r_{i}}\right|^{2} \quad i=1,2, \ldots, n
$$


and observe that the $X_{(i)}$ s are no longer independent. Rather, owing to (2) they constitute an order statistics; for the notation employed, $X_{(1)}$ is the largest order statistic, $X_{(n)}$ is the smallest.

The receiver first attempts to decode the strongest signal. If the decoding process is successful, the receiver removes the first strongest signal and then proceeds to decode the second strongest. For the base station to decode the message from the $j$-th strongest user $\mathrm{UE}_{(j)}$, the $j-1$ received signals with the strongest power have to be successfully recovered and removed first.

Recalling Shannon's capacity theorem, the achievable data rate of $\mathrm{UE}_{(j)}$ is

$$
R_{(j)}=\log _{2}\left(1+\frac{X_{(j)}}{\sum_{i=j+1}^{n} X_{(i)}+\sigma^{2}}\right) \quad \text { bits } / \mathrm{s} / \mathrm{Hz},
$$

for $j=1,2, \ldots, n-1$, where $\sigma^{2}$ is the noise power, and

$$
R_{(n)}=\log _{2}\left(1+\frac{X_{(n)}}{\sigma^{2}}\right) \quad \text { bits/s/Hz }
$$

for the last user $\mathrm{UE}_{(n)}$, whose received power is the weakest.

Denote by $\hat{R}_{(j)}$ the target data rate of $\mathrm{UE}_{(j)}$ and define the outage probability $P_{\text {out }}^{(j)}, j=1,2, \ldots$, $n$, as the probability that the SIC receiver can successfully recover the first strongest signal, the second strongest, up to the $j-1$, but it fails to decode the $j$-th strongest and all the subsequent signals. Analytically,

$$
P_{\text {out }}^{(j)}=1-P\left\{R_{(j)} \geq \hat{R}_{(j)}\right\}
$$

If we indicate by $\mathcal{E}_{k}, k=1,2, \ldots, n-1$, the random event identified by the condition

$$
\frac{X_{(k)}}{\sum_{i=k+1}^{n} X_{(i)}+\sigma^{2}} \geq \hat{\gamma}_{k}
$$

where

$$
\hat{\gamma}_{k}=2^{\hat{R}_{(k)}}-1, \quad k=1,2, \ldots, n,
$$

and by $\mathcal{E}_{n}$ the event in which the condition

$$
\frac{X_{(n)}}{\sigma^{2}} \geq \hat{\gamma}_{n}
$$

holds, then (6) is equivalently re-written as

$$
P_{\text {out }}^{(j)}=1-P\left\{\cap_{k=1}^{j} \mathcal{E}_{k}\right\}, \quad j=1,2, \ldots, n,
$$

where it is observed that the random events $\mathcal{E}_{1}, \mathcal{E}_{1}, \ldots, \mathcal{E}_{n}$ are statistically dependent. 
Indicate by $f_{\text {joint }_{n}}\left(x_{(1)}, x_{(2)}, \ldots, x_{(n)}\right)$ the joint probability density function (pdf) of the ordered set of random variables $X_{(i)}, i=1,2, \ldots, n$, and by $\mathcal{D}_{j}$ the region of the $X_{(1)}, X_{(2)}, \ldots, X_{(n)}$ space identified by the conditions:

$$
\mathcal{D}_{j}=\left\{\begin{array}{l}
X_{(1)} \geq \hat{\gamma_{1}} \cdot\left(\sum_{i=2}^{n} X_{(i)}+\sigma^{2}\right) \\
X_{(2)} \geq \hat{\gamma_{2}} \cdot\left(\sum_{i=3}^{n} X_{(i)}+\sigma^{2}\right) \\
\vdots \\
X_{(j)} \geq \hat{\gamma}_{j} \cdot\left(\sum_{i=j+1}^{n} X_{(i)}+\sigma^{2}\right) \\
0 \leq X_{(n)} \leq X_{(n-1)} \leq \cdots \leq X_{(2)} \leq X_{(1)}
\end{array} .\right.
$$

It follows that $P_{\text {out }}^{(j)}, j=1,2, \ldots, n$, is determined solving the integral

$$
P_{\text {out }}^{(j)}=1-\int \cdots \int_{\mathcal{D}_{j}} f_{\text {joint }_{n}}\left(x_{(1)}, x_{(2)}, \ldots, x_{(n-1)}, x_{(n)}\right) d x_{(n)} d x_{(n-1)} \ldots d x_{(2)} d x_{(1)} .
$$

Among the different outage probabilities $P_{\text {out }}^{(j)}$, observe that $P_{\text {out }}^{(1)}$ deserves a special place, as it coincides with the probability that the strongest signal cannot be correctly recovered; in this circumstance, not even one, out of the $n$ simultaneous transmissions, can be successfully decoded and power-domain NOMA fails. Indeed, the inequality $X_{(1)} \geq \hat{\gamma_{1}} \cdot\left(\sum_{i=2}^{n} X_{(i)}+\sigma^{2}\right)$ identifying the outage domain $\mathcal{D}_{1}$ represents the necessary condition for the SIC decoding process to begin. Equivalently stated, $P_{\text {out }}^{(1)}$ gives the probability that the adoption of power-based NOMA turns out detrimental, as not even the best signal is correctly decoded.

When evaluating $P_{\text {out }}^{(j)}$, the first non-trivial problem at hand is to determine $f_{\text {joint }_{n}}\left(x_{(1)}, x_{(2)}, \ldots, x_{(n)}\right)$. In this respect, let $f_{i}\left(x_{i}\right)$ be the pdf of the unordered random variable $X_{i}, i=1,2, \ldots, n$, defined in (1), whose pdf is available once the pdf of $h_{i}$ is known, as $p_{t, i}$ is a constant, and define $F_{n}$ as the following $n \times n$ matrix

$$
F_{n}=\left[\begin{array}{cccc}
f_{1}\left(x_{(1)}\right) & f_{2}\left(x_{(1)}\right) & \ldots & f_{n}\left(x_{(1)}\right) \\
f_{1}\left(x_{(2)}\right) & f_{2}\left(x_{(2)}\right) & \ldots & f_{n}\left(x_{(2)}\right) \\
\vdots & \vdots & \ddots & \vdots \\
f_{1}\left(x_{(n)}\right) & f_{2}\left(x_{(n)}\right) & \ldots & f_{n}\left(x_{(n)}\right)
\end{array}\right]
$$

where $f_{j}\left(x_{(i)}\right)$ denotes the pdf of the unordered random variable $X_{j}, j=1,2, \ldots, n$, when the function argument is the random sample $x_{(i)}$ of the ordered random variable $X_{(i)}$. For the purpose of what follows, recall that the permanent of a square matrix $A$, written as $\left.{ }_{\mid+}^{\mid} A\right|^{+}$, is defined like 
the determinant, except that all signs are positive. For an arbitrary $n$, it can be demonstrated that the joint pdf $f_{\text {joint }_{n}}\left(x_{(1)}, x_{(2)}, \ldots, x_{(n)}\right)$ of the ordered statistics $X_{(1)}, X_{(2)}, \ldots, X_{(n)}$ is

$$
f_{\text {joint }_{n}}\left(x_{(1)}, x_{(2)}, \ldots, x_{(n)}\right)=\left.\stackrel{+}{\left|F_{n}\right|}\right|^{+}
$$

where $F_{n}$ is given by (13). Last result is substantiated by the reasoning in [15], where the arguments of [16] are extended to prove the formulation in (14) with the use of permanents.

At first sight, (14) gives the impression that evaluating the integral in (12) might be quite cumbersome for an arbitrary value of $n$. However, the joint pdf obeys a highly peculiar structure, that allows a more convenient rewriting of it in the following terms: let $S_{i}=\left\{i_{1}, i_{2}, \ldots, i_{n}\right\}$ indicate the generic permutation of $\{1,2, \ldots, n\}$ in $\mathcal{S}_{\mathcal{N}}$. It follows that $f_{\text {joint }_{n}}\left(x_{(1)}, x_{(2)}, \ldots, x_{(n)}\right)$ can be equivalently written as

$$
f_{\text {joint }}\left(x_{(1)}, x_{(2)}, \ldots, x_{(n)}\right)=\sum_{S_{i} \in \mathcal{S}_{\mathcal{N}}} f_{1}\left(x_{\left(i_{1}\right)}\right) f_{2}\left(x_{\left(i_{2}\right)}\right) \ldots f_{n}\left(x_{\left(i_{n}\right)}\right) .
$$

Last expression highlights that the joint pdf exhibits the presence of $n$ ! terms, wherein the permutations of the arguments of the $f_{1}(\cdot), f_{2}(\cdot), \ldots, f_{n}(\cdot)$ pdfs appear. Replacing (15) in (12) leads to

$$
P_{\text {out }}^{(j)}=1-\int \cdots \int_{\mathcal{D}_{j}} \sum_{S_{i} \in \mathcal{S}_{\mathcal{N}}} f_{1}\left(x_{\left(i_{1}\right)}\right) f_{2}\left(x_{\left(i_{2}\right)}\right) \ldots f_{n}\left(x_{\left(i_{n}\right)}\right) d x_{(n)} d x_{(n-1)} \ldots d x_{(2)} d x_{(1)}
$$

and indicating by $I_{S_{i}}$ the result of the integral

$$
I_{S_{i}}=\int \ldots \int_{\mathcal{D}_{j}} g_{i_{1} i_{2} \ldots i_{n}}\left(x_{(1)}, x_{(2)}, \ldots, x_{(n)}\right) d x_{(n)} \ldots d x_{(1)}
$$

where

$$
g_{i_{1} i_{2} \ldots i_{n}}\left(x_{(1)}, x_{(2)}, \ldots, x_{(n)}\right)=f_{1}\left(x_{\left(i_{1}\right)}\right) f_{2}\left(x_{\left(i_{2}\right)}\right) \cdot \ldots \cdot f_{n}\left(x_{\left(i_{n}\right)}\right),
$$

then $P_{\text {out }}^{(j)}$ can be rewritten as:

$$
P_{\text {out }}^{(j)}=1-\sum_{S_{i} \in \mathcal{S}_{\mathcal{N}}} I_{S_{i}}, \quad \forall j, j=1,2, \ldots, n
$$

Luckily, when the random variables $X_{1}, X_{2}, \ldots, X_{n}$ obey the same statistical description, although with different mean values, for a permutation $S_{k}$ other than $S_{i}$, the $I_{S_{k}}$ term is obtained from $I_{S_{i}}$ through the analogous permutation of the $f_{i}(\cdot)$ 's arguments $x_{\left(i_{k}\right)}, k=1,2, \ldots, n$. That is to say, given the $n$-th fold integral in (17) has been solved once, e.g., $I_{S_{1}}$ has been determined for $S_{1}=\{1,2, \ldots, n\}$, then all the remaining $I_{S_{i}}$ terms are known. This significantly reduces $P_{\text {out }}^{(j)}$ computational complexity in $n$ regardless of the channel envelope statistics, i.e., no matter what pdf the random variables $h_{i}, i=1,2, \ldots, n$, obey to. 
Once $P_{\text {out }}^{(j)}$ has been obtained, the sum data rate that power-domain NOMA achieves is evaluated as:

$$
R_{N O M A}=\sum_{j=1}^{n} \hat{R}_{(j)} \cdot\left(1-P_{\text {out }}^{(j)}\right)
$$

B. $P_{\text {out }}^{(j)}$ Approximation for $j \geq 2$

The previous development highlighted how to reduce the complexity that hinders behind the exact analytical evaluation of the outage probability $P_{\text {out }}^{(j)}, j=1,2, \ldots, n$. The approach turns out particularly useful when evaluating $P_{\text {out }}^{(1)}$. As a matter of fact, next Section first considers the presence of Rayleigh fading and reports the exact closed-form expression of $P_{\text {out }}^{(1)}$ derived in the Appendix; the analytical expression is obtained applying the methodology illustrated in Subsection II-A, for an arbitrary value of $n$. Then, the simultaneous presence of Rayleigh fading and lognormal shadowing is examined, and an approximation is first introduced, to provide $P_{\text {out }}^{(1)}$ in closed-form, regardless of $n$, in this setting too.

Yet, when $j \geq 2$, the complexity in evaluating $P_{\text {out }}^{(j)}$ has also to be ascribed to an increasingly complex integration domain $\mathcal{D}_{j}$ in (11), as well as to the dependency among the events $\mathcal{E}_{1}, \mathcal{E}_{2}, \ldots, \mathcal{E}_{j}$. To alleviate the computational burden, this subsection explores the following approximation to $P_{\text {out }}^{(j)}, j \geq 2$ :

$$
P_{\text {out }}^{(j)} \approx 1-\prod_{k=1}^{j} P\left\{\mathcal{E}_{k}\right\}, \quad j \geq 2,
$$

that holds under the assumption that the random events $\mathcal{E}_{k}, k=1,2, \ldots, n$, be weakly dependent.

Beginning with $n=2$, recalling (7) and (9), $P_{\text {out }}^{(2)}$ specializes to

$$
P_{\text {out }}^{(2)} \approx 1-P\left\{\frac{X_{(1)}}{X_{(2)}+\sigma^{2}} \geq \hat{\gamma}_{1}\right\} \cdot P\left\{\frac{X_{(2)}}{\sigma^{2}} \geq \hat{\gamma}_{2}\right\} ;
$$

it is easy to recognize that the first term in the product on the right-hand side of (22) coincides with $1-P_{\text {out }}^{(1)}$. Moreover, indicating by $G_{(2)}(\cdot)$ the Cumulative Distribution Function $(\mathrm{CDF})$ of the random variable $X_{(2)}$, (22) is equivalently re-written as

$$
P_{\text {out }}^{(2)} \approx 1-\left(1-P_{\text {out }}^{(1)}\right) \cdot\left(1-G_{(2)}\left(\hat{\gamma}_{2} \sigma^{2}\right)\right) .
$$

With no loss in generality, let the unordered random variables $X_{i}, i=1,2, \ldots, n$, be numbered in accordance to the descending order of their mean received powers, so that $\bar{X}_{1}>\bar{X}_{2}>\ldots>\bar{X}_{n}$. Moreover, let us assume that the random variable $\delta_{i}$ measuring the spacing between $X_{i}$ and $X_{i-1}$, $\delta_{i}=\left|X_{i}-X_{i-1}\right|, i=2, \ldots, n$, takes on large values with probability close to 1 . In the scenarios 
where uplink NOMA is profitably employed, such approximation is verified, i.e., the spacing $\delta_{i}$ is sufficiently wide; as a matter of fact, this is the condition that allows to better discriminate among simultaneously received signals. Given this assumption holds, observe that it is possible to leverage upon a further approximation, namely, $G_{(2)}(\cdot)$ that appears in (23) is replaced by $G_{2}(\cdot)$, the CDF of the unordered random variable $X_{2}$, leading to

$$
P_{\text {out }}^{(2)} \approx 1-\left(1-P_{\text {out }}^{(1)}\right) \cdot\left(1-G_{2}\left(\hat{\gamma}_{2} \sigma^{2}\right)\right)
$$

that represents the final, approximated $P_{\text {out }}^{(2)}$ expression when $n=2$.

When $n=3$,

$$
P_{\text {out }}^{(2)} \approx 1-P\left\{\frac{X_{(1)}}{X_{(2)}+X_{(3)}+\sigma^{2}} \geq \hat{\gamma}_{1}\right\} \cdot P\left\{\frac{X_{(2)}}{X_{(3)}+\sigma^{2}} \geq \hat{\gamma}_{2}\right\}=1-\left(1-P_{\text {out }}^{(1)}\right) \cdot\left(1-P_{\text {out }_{2,3}}^{(1)}\right)
$$

wherein $P_{\text {out }_{2,3}}^{(1)}$ is the probability that NOMA fails in the only presence of the second and third strongest users. As regards $P_{\text {out }}^{(3)}$, the same approximation leveraged in (24) leads to

$$
P_{\text {out }}^{(3)} \approx 1-\left(1-P_{\text {out }}^{(1)}\right) \cdot\left(1-P_{\text {out }_{2,3}}^{(1)}\right) \cdot\left(1-G_{3}\left(\hat{\gamma}_{3} \sigma^{2}\right)\right),
$$

$G_{3}(\cdot)$ being the CDF of the unordered random variable $X_{3}$.

For a generic $j, P_{\text {out }}^{(j)}$ approximated expression is therefore provided by

$$
P_{\text {out }}^{(j)} \approx \begin{cases}1-\left(1-P_{\text {out }}^{(1)}\right) \cdot \prod_{h=1}^{j-1}\left(1-P_{\text {out }_{h+1, h+2, \ldots, n}}^{(1)}\right) & j<n \\ 1-\left(1-P_{\text {out }}^{(1)}\right) \cdot \prod_{h=1}^{n-1}\left(1-P_{\text {out }_{h+1, h+2, \ldots, n}}^{(1)}\right) \cdot\left(1-G_{n}\left(\hat{\gamma}_{n} \sigma^{2}\right)\right) & j=n\end{cases}
$$

where $P_{\text {out }_{h+1, h+2, \ldots, n}}^{(1)}$ is the probability that NOMA fails in the only presence of the $(h+1)$-th, $(h+2)$-th, $\ldots, n$-th strongest users, and $G_{n}(\cdot)$ is the CDF of the $n$-th random variable $X_{n}$, i.e., the CDF of the power received from the $n$-th UE, the most distant user from the base, equipped with the weakest average received power.

Provided the approximation holds, last expression is illuminating, as it reveals that: (i) the outage probability $P_{\text {out }}^{(j)}, j \geq 2$, depends on $P_{\text {out }}^{(1)}$, the probability that NOMA fails in the presence of the same number of users; (ii) moreover, $P_{\text {out }}^{(j)}$ can be readily computed, given the expression of the probability $P_{\text {out }}^{(1)}$ that NOMA fails in the presence of $n, n-1, \ldots, n-j+1$ users is known. Also observe that the expressions we have obtained can be exploited for different fading descriptions.

The Numerical Results Section will highlight that it is possible to rely upon the proposed approximation of $P_{o u t}^{(j)}, j \geq 2$, in several meaningful settings. To the authors' knowledge, there is however no means to conclude whether (27) provides an upper or lower bound to the outage. 
As previously anticipated, when Rayleigh fading is considered, next Section will report the exact analytical expression of $P_{\text {out }}^{(1)}$ and the closed-form approximation of $P_{o u t}^{(j)}, j \geq 2$, for an arbitrary number of simultaneously transmitting UEs. When shadowing is also introduced, the closed-form $P_{\text {out }}^{(j)}$ approximation will be provided for $j \geq 1$.

\section{FADING MODELS}

\section{A. Rayleigh Fading}

When the presence of Rayleigh fading is assumed, the probability density function (pdf) of the received signal power $X_{i}$ is exponential:

$$
f_{i}\left(x_{i}\right)=\frac{1}{\bar{X}_{i}} \exp \left(\frac{-x_{i}}{\bar{X}_{i}}\right)
$$

with mean $\bar{X}_{i}$,

$$
\bar{X}_{i}=p_{t, i} \cdot k_{p} D_{i}^{-\alpha}
$$

$k_{p}$ being a constant that depends on the operating frequency and $D_{i}$ the distance between $\mathrm{UE}_{i}$ and the base station.

In Appendix A, it is proved that when $\hat{\gamma}_{1} \geq 1, P_{\text {out }}^{(1)}$ for an arbitrary number of users $n$ obeys the expression:

$$
P_{\text {out }}^{(1)}=1-\sum_{k=1}^{n} \frac{\exp \left(\frac{-\hat{\gamma}_{1} \sigma^{2}}{\bar{X}_{k}}\right)}{\prod_{\substack{i=1 \\ i \neq k}}^{n}\left(1+\frac{\bar{X}_{i}}{\bar{X}_{k}} \hat{\gamma}_{1}\right)}
$$

that immediately reveals what limits NOMA faces, if the number $n$ of simultaneously active users is increased from 2 to higher values.

In turn, taking advantage of (30), the fading-independent approximation of $P_{\text {out }}^{(j)}$ provided by (27), $j \geq 2$, specializes to 


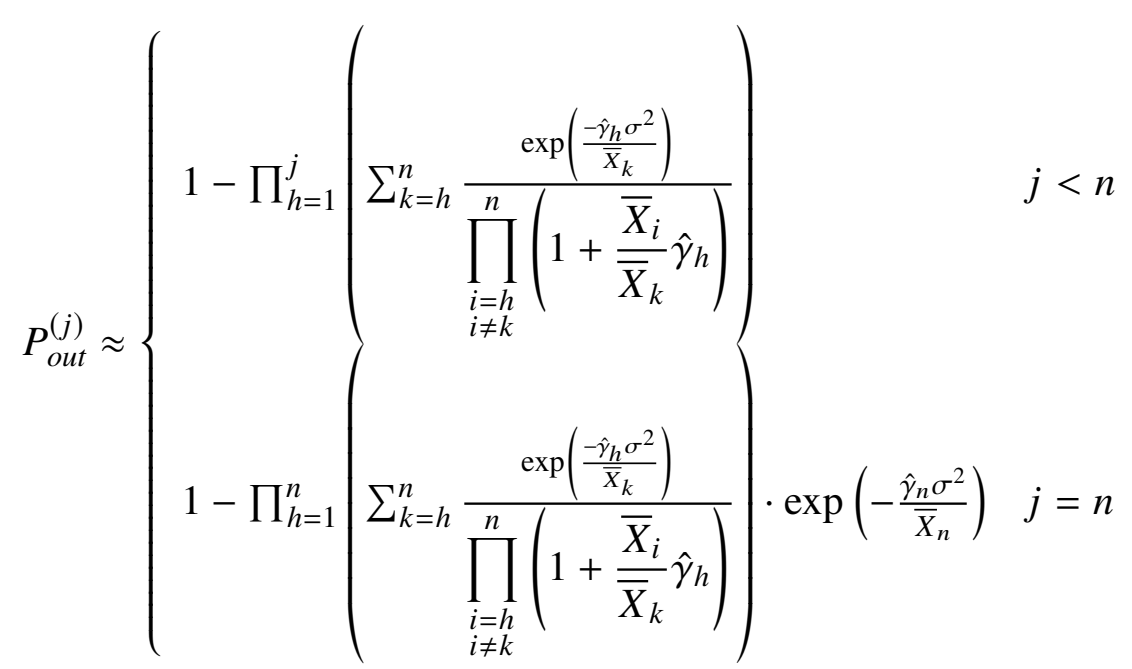

The constraint on $\hat{\gamma}_{1}$ deserves a careful remark: it is the authors' belief that it does not represent a limiting factor, rather, a fairly widespread requirement in upcoming settings. As an example, high-end industrial IoT use cases are expected to require conspicuous data rates, exceeding the system available bandwidth, to support video-assisted services ranging from process monitoring to augmented video-reality [1].

\section{B. Rayleigh-lognormal Shadowed Fading}

When the envelope of the received signal is subject to both Rayleigh fading and lognormal shadowing, the $X_{i}$ pdf is

$$
f_{i}\left(x_{i}\right)=\int_{0}^{+\infty} \frac{1}{\bar{x}} \exp \left(\frac{-x_{i}}{\bar{x}}\right) \frac{1}{\sqrt{2 \pi} \frac{\sigma_{L}}{h} \bar{x}} \exp \left(-\frac{\left(\ln (\bar{x})-\mu_{i}\right)^{2}}{\frac{2 \sigma_{L}^{2}}{h^{2}}}\right) d \bar{x}
$$

where $\sigma_{L}$ is the standard deviation of the Gaussian random variable modeling lognormal shadowing in $\mathrm{dB}, \mu_{i}$ depends on the distance attenuation law, $\mu_{i}=\ln \left(p_{t, i} \cdot k_{p} D_{i}^{-\alpha}\right)$, and $h=\frac{10}{\ln (10)}$. In our analysis, $\sigma_{L}$ is assumed to be the same for all signals.

An additional hurdle is present here, because of the integral in (32). We therefore propose an approximation to (32), exploiting the approach put forth by Holtzman in [17]. According to [17], given a function $\psi(\theta)$ of a Gaussian random variable $\theta$ with mean $\mu_{\theta}$ and variance $\sigma_{\theta}^{2}$, the expectation $\mathbb{E}[\psi(\theta)]$ can be approximated by

$$
\mathbb{E}[\psi(\theta)] \approx \frac{2}{3} \psi\left(\mu_{\theta}\right)+\frac{1}{6} \psi\left(\mu_{\theta}+\sqrt{3} \sigma_{\theta}\right)+\frac{1}{6} \psi\left(\mu_{\theta}-\sqrt{3} \sigma_{\theta}\right) .
$$

For the examined case, it is observed that the change of variable

$$
y=h \cdot \ln (\bar{x})
$$


that is, $\bar{x}=\exp \left(\frac{y}{h}\right)$, leads to rewrite (32) in the form

$$
f_{i}\left(x_{i}\right)=\int_{-\infty}^{+\infty} \frac{1}{\exp \left(\frac{y}{h}\right)} \exp \left(\frac{-x_{i}}{\exp \left(\frac{y}{h}\right)}\right) \frac{1}{\sqrt{2 \pi} \sigma_{L}} \exp \left(-\frac{\left(y-\mu_{i}\right)^{2}}{2 \sigma_{L}^{2}}\right) d y
$$

that can therefore be approximated as:

$$
f_{i}\left(x_{i}\right) \approx \sum_{k=1}^{3} \frac{a_{k}}{b_{i, k}} \exp \left(\frac{-x_{i}}{b_{i, k}}\right)
$$

where $a_{1}=\frac{2}{3}, a_{2}=a_{3}=\frac{1}{6}, b_{i, 1}=\exp \left(\frac{\mu_{i}}{h}\right), b_{i, 2}=\exp \left(\frac{\left(\mu_{i}+\sqrt{3} \sigma_{L}\right)}{h}\right)$ and $b_{i, 3}=\exp \left(\frac{\left(\mu_{i}-\sqrt{3} \sigma_{L}\right)}{h}\right)$.

This linear combination of exponential functions allows to leverage the results obtained in the previous case of Rayleigh fading. Hence, when lognormal shadowing is added, for $n$ superimposed signals $P_{\text {out }}^{(1)}$ is approximated by

$$
P_{\text {out }}^{(1)} \approx 1-\sum_{k_{1}=1}^{3} a_{k_{1}} \cdot \ldots \cdot \sum_{k_{n-1}=1}^{3} a_{k_{n-1}} \sum_{k_{n}=1}^{3} a_{k_{n}}\left[\sum_{i=1}^{n} \frac{\exp \left(\frac{-\hat{\gamma}_{1} \sigma^{2}}{b_{i, k_{i}}}\right)}{\prod_{\substack{j=1 \\ j \neq i}}^{n}\left(1+\frac{b_{j, k_{j}}}{b_{i, k_{i}}} \hat{\gamma}_{1}\right)}\right]
$$

and $P_{\text {out }}^{(j)}, j \geq 2$, follows from (27) and it is approximated by

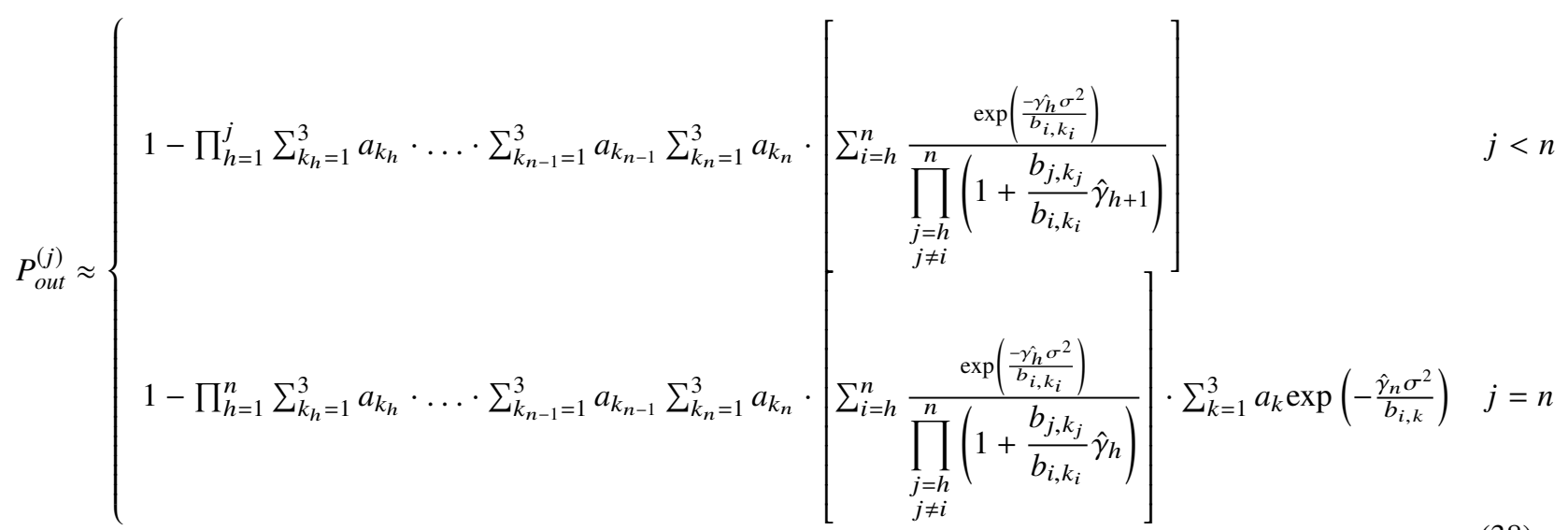

In next Section, the excellent accuracy of the approximations in (31), (37) and (38) will be demonstrated for several choices of system parameters.

\section{NumERicAl RESUlts}

An exemplary set of numerical results is reported next, in order to highlight the accuracy of the proposed analytical approaches, as well as to provide useful insights on the uplink performance of power-domain NOMA, when the dynamic-ordered SIC receiver is employed. 


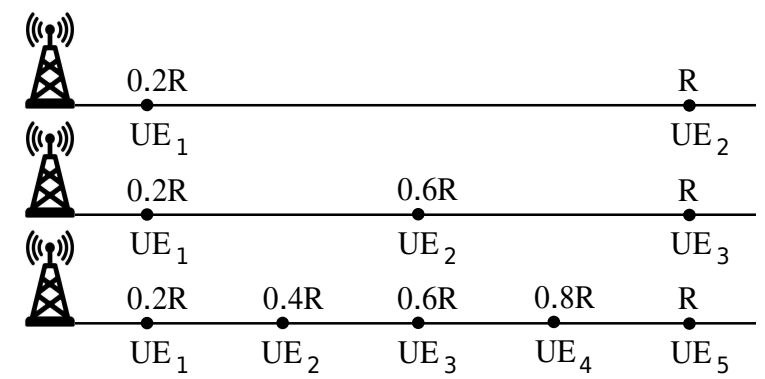

Fig. 1. Users location along the cell radius when $n=2,3,5$

The results have been obtained for the following configuration: in (6), the target data rate $\hat{R}_{(j)}$ is set to $1.2 \mathrm{bits} / \mathrm{s} / \mathrm{Hz}, \forall j, j=1,2, \ldots, n$; in (29), the $k_{p}$ constant is $\left(\frac{c}{4 \pi f_{c}}\right)$, where $c$ is the speed of light and omnidirectional antennas are assumed. The operating frequency is $f_{c}=2 \mathrm{GHz}$, the pathloss exponent is $\alpha=2$ and the cell radius is $R=1000 \mathrm{~m}$. As regards lognormal shadowing, unless otherwise stated, in (32) $\sigma_{L}=4 \mathrm{~dB}$. To improve the base station capability to recover the signals coming from distinct UEs, the transmitted powers are set so as to attribute higher power levels to UEs closer to the base station. Unless otherwise stated, the UEs location along the cell radius is the one illustrated in Fig. 1. Namely, the ratio between the transmitted powers of $\mathrm{UE}_{i}$ and $\mathrm{UE}_{j}$, with distances $D_{i}$ and $D_{j}$ from the base, $D_{i}<D_{j}$, is set to

$$
\frac{p_{t, i}}{p_{t, j}}=10^{\frac{(j-i) \Delta}{10}}
$$

and the power back-off step is $\Delta=6 \mathrm{~dB}$.

In the first set of figures, the outage probabilities are reported as a function of the largest average received Signal-to-Noise Ratio (SNR). Recalling that the UEs are indexed so that $\bar{X}_{1}>$ $\bar{X}_{2}>\cdots>\bar{X}_{n}$, it follows that SNR $=\bar{X}_{1} / \sigma^{2}$. In other words, as the power law assignment privileges users that are closer to the base, the SNR is the average received signal-to-noise ratio of the UE that is the nearest to the base station.

When Rayleigh fading is considered, Fig. 2 shows $P_{\text {out }}^{(1)}$ as a function of the SNR, if $n=2,3$, 5 users are simultaneously transmitting. As indicated in Fig. 2, when $n=2$, the distance $D_{1}$ of $\mathrm{UE}_{1}$ from the base is $0.2 R$ and the distance $D_{2}$ of $\mathrm{UE}_{2}$ from it is $R$; when $n=3, D_{1}=0.2 R$, $D_{2}=0.6 R$ and $D_{3}=R$; when $n=5, D_{1}=0.2 R, D_{2}=0.4 R, D_{3}=0.6 R, D_{4}=0.8 R$ and $D_{5}=R$. Solid lines refer to the exact analytical evaluation, markers to $P_{\text {out }}^{(1)}$ values determined through Monte Carlo simulation, considering $10^{5}$ samples for each plotted value. The perfect match between the analytical and the simulation results confirms the correctness of the exact 


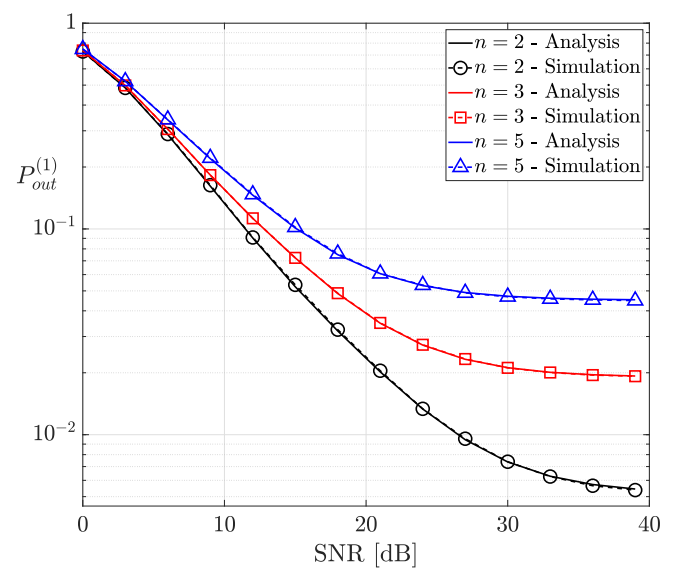

Fig. 2. Rayleigh fading: $P_{\text {out }}^{(1)}$ as a function of the SNR, $n=2,3,5$

closed-form of $P_{\text {out }}^{(1)}$ provided by (30). The figure also indicates that $P_{\text {out }}^{(1)}$ is close to 1 when the SNR is smaller than $5 \mathrm{~dB}$, regardless of $n$. As a matter of fact, in this low SNR region, the achievable data rate of the strongest user is limited by the weak level of the received signal, rather than by the presence of simultaneously transmitting users. As the SNR increases above $10 \mathrm{~dB}$, the impact of a larger number of interfering users gradually becomes more evident. Yet, observe that for $n=5, P_{\text {out }}^{(1)}$ is always below $1 \%$ for all SNR values in the $[15,+\infty]$ range.

Fig. 3 shows $P_{\text {out }}^{(1)}$ as a function of the SNR, when the received signal is subject to both Rayleigh fading and log-normal shadowing, and it reveals that the closed-form in (37) is an

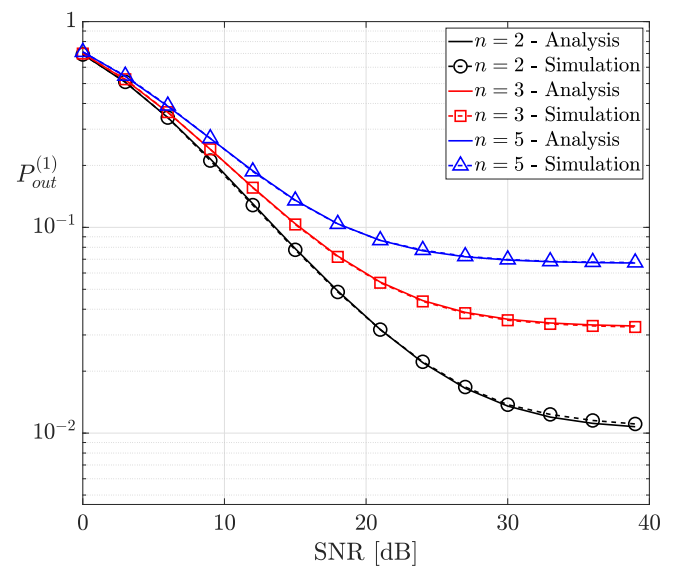

Fig. 3. Rayleigh fading and log-normal shadowing: $P_{\text {out }}^{(1)}$ as a function of the SNR, $n=2,3,5$ 


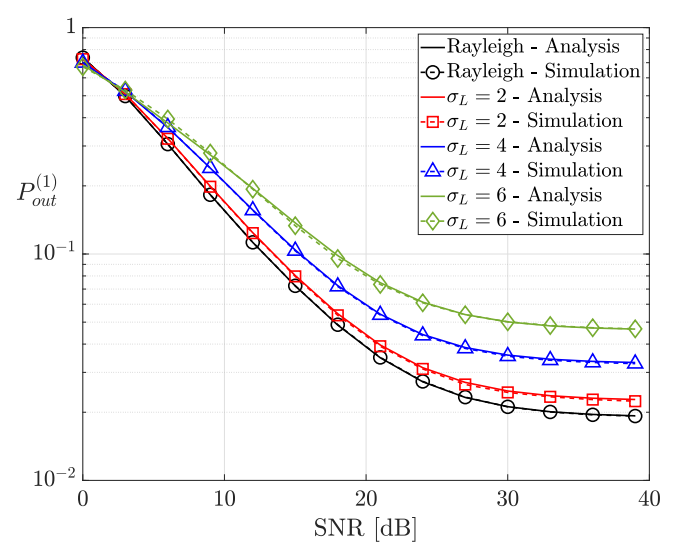

Fig. 4. $P_{\text {out }}^{(1)}$, Rayleigh fading and Rayleigh plus lognormal, $n=3$

excellent approximation to the exact $P_{\text {out }}^{(1)}$ computed via simulation. In this scenario too, the impact on $P_{\text {out }}^{(1)}$ of a larger number of users can be appreciated only for SNR values greater than $10 \mathrm{~dB}$. The relative position of the curves is the same as observed in Fig. 2. However, $P_{\text {out }}^{(1)}$ takes on higher values than in the presence of Rayleigh fading only. For instance, when SNR $=30$ $\mathrm{dB}$, for $n=2 P_{\text {out }}^{(1)}$ increases from $7 \times 10^{-3}$ determined in the presence of Rayleigh fading to $1.4 \times 10^{-2}$ when lognormal shadowing is also taken into account, and raises from $4.7 \times 10^{-2}$ to $6.9 \times 10^{-2}$ for $n=5$. This indicates that the shadowing plays a non-negligible role in the outage probability evaluation.

To better understand the influence of lognormal shadowing, Fig. 4 compares $P_{\text {out }}^{(1)}$ in the presence of Rayleigh fading against $P_{\text {out }}^{(1)}$ in the additional presence of lognormal shadowing, when $n=3$ and three different values of $\sigma_{L}$, namely, 2, 4 and $6 \mathrm{~dB}$ are considered. The black lowest curve and the circle markers refer to the benchmark case of Rayleigh fading; the red, blue and green curves, paired with the square, triangle and diamond markers, respectively, refer to the Rayleigh plus lognormal case. The results corroborate what was previously anticipated, quantifying the remarkable impact of the shadowing on $P_{\text {out }}^{(1)}$ for increasing values of $\sigma_{L}$. For instance, when $\sigma_{L}=6 \mathrm{~dB}$ and SNR $>30 \mathrm{~dB}, P_{\text {out }}^{(1)}$ is 2.4 times larger than for the case of Rayleigh fading only. Also note the tightness of the approximation provided by (37): here too, the results obtained by simulation are nearly undistinguishable from the analytical outcomes, no matter what $\sigma_{L}$ value is examined.

Next, Fig. 5a and 5b report $P_{\text {out }}^{(1)}, P_{\text {out }}^{(2)}$ and $P_{\text {out }}^{(3)}$ for the case of Rayleigh fading and Rayleigh 


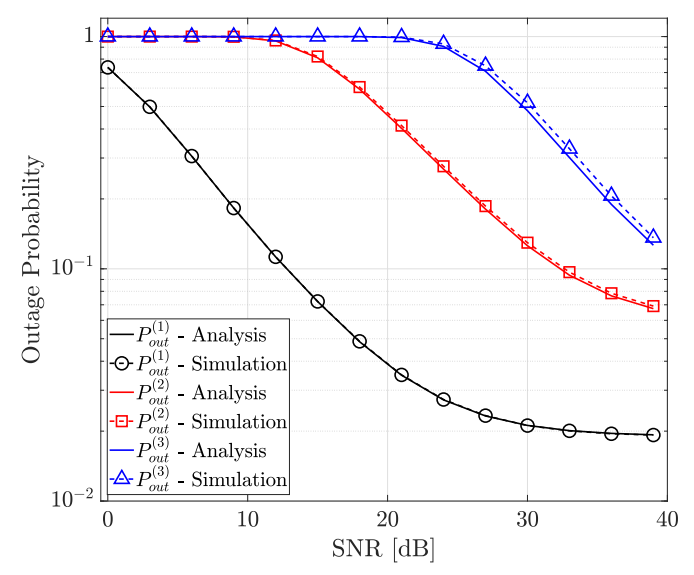

(a) Rayleigh Fading

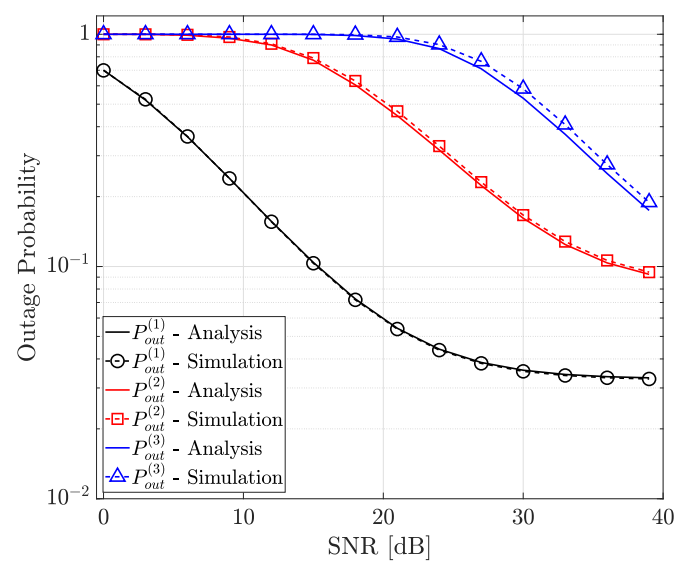

(b) Rayleigh fading plus log-normal shadowing

Fig. 5. Outage probability vs SNR, $n=3$

plus lognormal shadowing, respectively, when $n=3$. As regards $P_{\text {out }}^{(2)}$ and $P_{\text {out }}^{(3)}$, these figure show the impressive accuracy of the approximation proposed in Section III and detailed in eqs.(31) and (38). Furthermore, they reveal that $P_{\text {out }}^{(2)}$ and $P_{\text {out }}^{(3)}$ take on significantly high values, with and without lognormal shadowing. As expected, $P_{\text {out }}^{(3)}$ takes on the worst values, as the signal coming from the third strongest user can be decoded only if both the second and the first strongest signals have already been decoded.

When considering both Rayleigh fading and lognormal shadowing, an alternative view is provided by Fig.6, that shows $P_{\text {out }}^{(1)}, P_{\text {out }}^{(2)}$ and $P_{\text {out }}^{(3)}$ as a function of $D_{2} / R$, the normalized distance of $\mathrm{UE}_{2}$ from the base, when $\mathrm{UE}_{1}$ and $\mathrm{UE}_{3}$ distances are $D_{1}=0.2 R$ and $D_{3}=R$, respectively. This figure indicates that the values of $P_{\text {out }}^{(2)}$ and $P_{\text {out }}^{(3)}$ lie in the range of a few percentage points; 


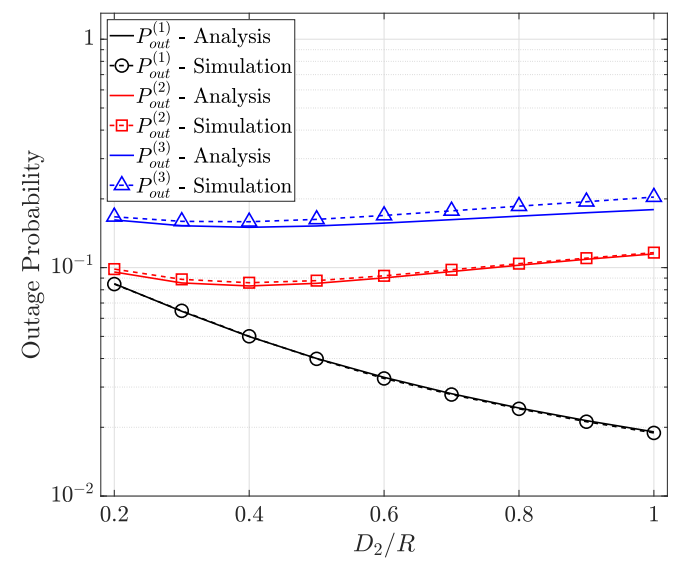

Fig. 6. Outage probability as a function of $D_{2} / R, n=3, \mathrm{SNR}=30 \mathrm{~dB}$

equivalently stated, approximately in $90 \%$ of the cases it is possible to support 2 simultaneous communications (and in $80 \%$ of the cases even 3). If the involved UEs all require maximum reliability, this is unacceptable. Yet, resorting to power-domain NOMA becomes truly interesting in alternative settings: for instance, whenever communication redundancy can be introduced without an excessive overhead, as it happens when a modest number of packet re-transmissions are introduced and packets exhibit a modest size. The figure also shows that $P_{\text {out }}^{(2)}$ and $P_{\text {out }}^{(3)}$ minima lie at $D_{2}=0.4 R$ and that they are not so critical, revealing that the location of the UEs does not have to be identified with extreme accuracy. Also observe that the tightness of the proposed approximation in evaluating $P_{\text {out }}^{(3)}$ slightly worsens as $D_{2}$ approaches $D_{3}$; this happens since the spacing $\delta_{3}$ no longer verifies the assumption of taking on large values with probability close to 1.

Last, Fig. 7 displays $R_{N O M A}$, the sum data rate of power-domain NOMA of (20), as a function of the SNR in the simultaneous presence of Rayleigh fading and lognormal shadowing, when $n=2$ and $n=3$, and compares it against the OMA data rate. The latter scheme is examined under the hypothesis that the signal-to-noise ratio of the OMA user coincides with SNR, the signal-to-noise ratio of the NOMA UE that is the nearest to the base station. Here too, the accuracy of the proposed approximation is striking. The gain of NOMA over OMA becomes more and more evident for increasing SNR values. Moreover, at high SNR regimes the NOMA system with $n=3$ users achieves a sum data rate significantly greater than $2.4 \mathrm{bits} / \mathrm{s} / \mathrm{Hz}$, the maximum data rate NOMA attains when $n=2$. 


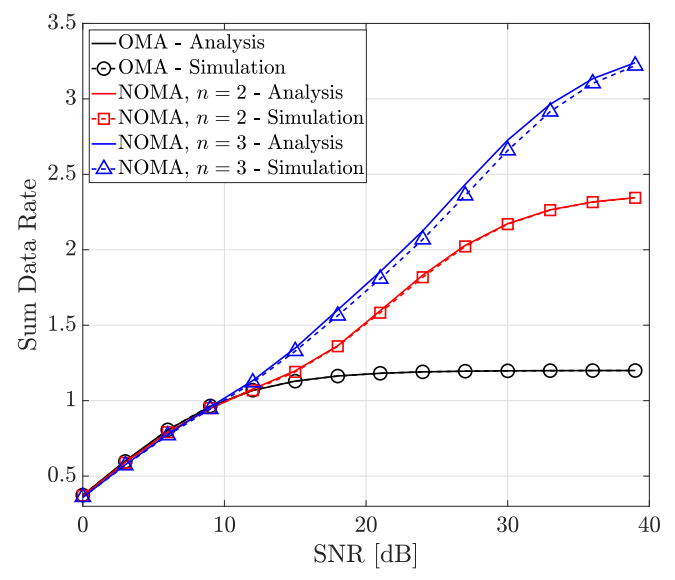

Fig. 7. Sum data rate of OMA and NOMA as a function of SNR, Rayleigh and lognormal shadowing

\section{CONCLUSIONS}

This work has proposed a novel analytical approach to evaluate the outage probabilities of uplink power-domain NOMA, when a dynamic-ordered SIC receiver is employed. The method has been employed in the presence of Rayleigh fading, and Rayleigh plus lognormal shadowing. In the former setting, it has allowed to determine the probability that NOMA fails through an exact analytical expression, for a generic number of superimposed signals; in the second examined scenario, such probability has been obtained in closed-form via an excellent approximation. Moreover, the current study has put forth an approximated expression of the probability that the SIC receiver does not succeed in decoding the second, third, $n$-th strongest user. Monte Carlo simulations have demonstrated the accuracy of the results obtained through the proposed approximations, that clearly quantify the effects of an increasing number of simultaneous users on system performance. The analysis has also disclosed to what extent lognormal shadowing affects NOMA behavior, revealing that its presence significantly deteriorates performance with respect to the case of Rayleigh fading only.

\section{APPENDIX A}

\section{DERIVATION OF $P_{\text {out }}^{(1)}$ FOR AN ARBITRARY NUMBER OF USERS}

In the presence of Rayleigh fading, the outage probability $P_{\text {out }}^{(1)}$ is evaluated beginning with the special case $n=2$. From (19), $P_{\text {out }}^{(1)}$ specializes to

$$
P_{\text {out }}^{(1)}=1-\left(I_{S_{1}}+I_{S_{2}}\right)
$$


where $S_{1}=\{1,2\}, S_{2}=\{2,1\}$,

$$
I_{S_{1}}=\iint_{\mathcal{D}_{1}} f_{1}\left(x_{(1)}\right) f_{2}\left(x_{(2)}\right) d x_{(2)} d x_{(1)}=\iint_{\mathcal{D}_{1}} \frac{1}{\bar{X}_{1}} \exp \left(-\frac{x_{(1)}}{\bar{X}_{1}}\right) \cdot \frac{1}{\bar{X}_{2}} \exp \left(-\frac{x_{(2)}}{\bar{X}_{2}}\right) d x_{(2)} d x_{(1)}
$$

and $\mathcal{D}_{1}$ is

$$
\mathcal{D}_{1}=\left\{\begin{array}{l}
X_{(1)} \geq \hat{\gamma_{1}} \cdot\left(X_{(2)}+\sigma^{2}\right) \\
X_{(1)} \geq X_{(2)} \geq 0
\end{array} .\right.
$$

When the target data rate of the strongest user $\hat{\gamma}_{1}$ is at least equal to $1 \mathrm{bit} / \mathrm{s} / \mathrm{Hz}$, solving the integral in (41) gives

$$
I_{S_{1}}=\frac{\exp \left(\frac{-\hat{\gamma}_{1} \sigma^{2}}{\bar{X}_{1}}\right)}{1+\frac{\bar{X}_{2}}{\bar{X}_{1}} \hat{\gamma}_{1}} .
$$

From (43), $I_{S_{2}}$ is readily determined as

$$
I_{S_{2}}=\frac{\exp \left(\frac{-\hat{\gamma}_{1} \sigma^{2}}{\bar{X}_{2}}\right)}{1+\frac{\bar{X}_{1}}{\bar{X}_{2}} \hat{\gamma}_{1}}
$$

and $P_{\text {out }}^{(1)}$ follows:

$$
P_{\text {out }}^{(1)}=1-\left(\frac{\exp \left(\frac{-\hat{\gamma}_{1} \sigma^{2}}{\bar{X}_{1}}\right)}{1+\frac{\bar{X}_{2}}{\bar{X}_{1}} \hat{\gamma}_{1}}+\frac{\exp \left(\frac{-\hat{\gamma}_{1} \sigma^{2}}{\bar{X}_{2}}\right)}{1+\frac{\bar{X}_{1}}{\bar{X}_{2}} \hat{\gamma}_{1}}\right) .
$$

When $n=3, P_{\text {out }}^{(1)}$ exhibits 3 ! distinct contributions. The first of them, $I_{S_{1}}, S_{1}=\{1,2,3\}$, is

$$
I_{S_{1}}=\iiint_{\mathcal{D}_{1}} f_{1}\left(x_{(1)}\right) f_{2}\left(x_{(2)}\right) f_{3}\left(x_{(3)}\right) d x_{(3)} d x_{(2)} d x_{(1)}
$$

where $\mathcal{D}_{1}$ is identified by the conditions

$$
\mathcal{D}_{1}=\left\{\begin{array}{l}
X_{(1)} \geq \hat{\gamma_{1}} \cdot\left(X_{(2)}+X_{(3)}+\sigma^{2}\right) \\
X_{(1)} \geq X_{(2)} \geq X_{(3)} \geq 0
\end{array} .\right.
$$

So,

$$
\begin{aligned}
& I_{S_{1}}=\iiint_{\mathcal{D}_{1}} p_{123}\left(x_{(1)}, x_{(2)}, x_{(3)}\right) d x_{(3)} d x_{(2)} d x_{(1)} \\
& =\iiint_{\mathcal{D}_{1}} \frac{1}{\bar{X}_{1}} \exp \left(-\frac{x_{(1)}}{\bar{X}_{1}}\right) \cdot \frac{1}{\bar{X}_{2}} \exp \left(-\frac{x_{(2)}}{\bar{X}_{2}}\right) \cdot \frac{1}{\bar{X}_{3}} \exp \left(-\frac{x_{(3)}}{\bar{X}_{3}}\right) d x_{(3)} d x_{(2)} d x_{(1)} .
\end{aligned}
$$

After a few lengthy steps, last integral is solved and leads to the following result

$$
I_{S_{1}}=\frac{\exp \left(\frac{-\hat{\gamma_{1}} \sigma^{2}}{\overline{X_{1}}}\right){\overline{X_{1}}}^{2} \overline{X_{2}}}{\left(\overline{X_{1}}+\overline{X_{2}} \hat{\gamma_{1}}\right)\left(\overline{X_{1} X_{2}}+\overline{X_{1} X_{3}} \hat{\gamma_{1}}+\overline{X_{2} X_{3}} \hat{\gamma_{1}}+\overline{X_{2} X_{3}}{\hat{\gamma_{1}}}^{2}\right)}
$$


that more aptly is written as

$$
I_{S_{1}}=\frac{\exp \left(\frac{-\hat{\gamma_{1}} \sigma^{2}}{\bar{X}_{1}}\right)}{\left(1+\frac{\bar{X}_{2}}{\bar{X}_{1}} \hat{\gamma}_{1}\right)\left(1+\frac{\bar{X}_{3}}{\bar{X}_{2}} \hat{\gamma}_{1}+\frac{\bar{X}_{3}}{\bar{X}_{1}} \hat{\gamma}_{1}\left(\hat{\gamma}_{1}+1\right)\right)} .
$$

Once $I_{S_{1}}$ is determined, all the remaining contributions can be obtained permuting over $\mathcal{S}_{\mathcal{N}}$. On purpose, the set $S_{2}=\{1,3.2\}$ is considered next, which provides the result

$$
I_{S_{2}}=\frac{\exp \left(\frac{-\hat{\gamma}_{1} \sigma^{2}}{\bar{X}_{1}}\right) \bar{X}_{1}^{2} \bar{X}_{3}}{\left(\bar{X}_{1}+\bar{X}_{3} \cdot \hat{\gamma}_{1}\right)\left(\bar{X}_{1} \bar{X}_{2}+\bar{X}_{1}\left(\bar{X}_{3}+2 \bar{X}_{2} \bar{X}_{3} \hat{\gamma}_{1}\right)\right)} .
$$

Observe that, when the sum $I_{S_{1}}+I_{S_{2}}$ is computed, it gives

$$
C_{1}=I_{S_{1}}+I_{S_{2}}=\frac{\exp \left(\frac{-\hat{\gamma}_{1} \sigma^{2}}{\bar{X}_{1}}\right) \bar{X}_{1}^{2}}{\left(\bar{X}_{1}+\bar{X}_{2} \hat{\gamma}_{1}\right)\left(\bar{X}_{1}+\bar{X}_{3} \hat{\gamma}_{1}\right)}=\frac{\exp \left(\frac{-\hat{\gamma}_{1} \sigma^{2}}{\bar{X}_{1}}\right)}{\left(1+\frac{\bar{X}_{2}}{\bar{X}_{1}} \hat{\gamma}_{1}\right)\left(1+\frac{\bar{X}_{3}}{\bar{X}_{1}} \hat{\gamma_{1}}\right)} .
$$

If we now introduce the sets $S_{3}=\{2,1,3\}$ and $S_{4}=\{3,1,2\}$, permuting $\bar{X}_{1}, \bar{X}_{2}$ and $\bar{X}_{3}$ in (50) $I_{S_{3}}$ and $I_{S_{4}}$ are also determined. Their sum gives

$$
C_{2}=I_{S_{3}}+I_{S_{4}}=\frac{\exp \left(\frac{-\hat{\gamma}_{1} \sigma^{2}}{\bar{X}_{2}}\right) \bar{X}_{2}^{2}}{\left(\bar{X}_{2}+\bar{X}_{1} \hat{\gamma}_{1}\right)\left(\bar{X}_{2}+\bar{X}_{3} \hat{\gamma}_{1}\right)}=\frac{\exp \left(\frac{-\hat{\gamma}_{1} \sigma^{2}}{\bar{X}_{2}}\right)}{\left(1+\frac{\bar{X}_{1}}{\bar{X}_{2}} \hat{\gamma}_{1}\right)\left(1+\frac{\bar{X}_{3}}{\bar{X}_{2}} \hat{\gamma}_{1}\right)}
$$

The same applies to $S_{5}=\{3,2,1\}$ and $S_{6}=\{2,3,1\}$, whose sum $C_{3}$ is

$$
C_{3}=I_{S_{5}}+I_{S_{6}}=\frac{\exp \left(\frac{-\hat{\gamma}_{1} \sigma^{2}}{\bar{X}_{3}}\right) \bar{X}_{3}^{2}}{\left(\bar{X}_{3}+\bar{X}_{1} \hat{\gamma}_{1}\right)\left(\bar{X}_{3}+\bar{X}_{2} \hat{\gamma}_{1}\right)}=\frac{\exp \left(\frac{-\hat{\gamma}_{1} \sigma^{2}}{\bar{X}_{3}}\right)}{\left(1+\frac{\bar{X}_{1}}{\bar{X}_{3}} \hat{\gamma}_{1}\right)\left(1+\frac{\bar{X}_{2}}{\bar{X}_{3}} \hat{\gamma}_{1}\right)} .
$$

Therefore, in the presence of $n=3$ users and under the previous hypothesis $\hat{\gamma}_{1} \geq 1, P_{\text {out }}^{(1)}$ is amenable to the writing

$$
\begin{aligned}
& P_{\text {out }}^{(1)}=1-\sum_{S_{i} \in S_{N}} I_{S_{i}}=1-\sum_{k=1}^{3} C_{k}= \\
& =1-\left(\frac{\exp \left(\frac{-\hat{\gamma}_{1} \sigma^{2}}{\bar{X}_{1}}\right)}{\left(1+\frac{\bar{X}_{2}}{\bar{X}_{1}} \hat{\gamma}_{1}\right)\left(1+\frac{\bar{X}_{3}}{\bar{X}_{1}} \hat{\gamma}_{1}\right)}+\frac{\exp \left(\frac{-\hat{\gamma}_{1} \sigma^{2}}{\bar{X}_{2}}\right)}{\left(1+\frac{\bar{X}_{1}}{\bar{X}_{2}} \hat{\gamma}_{1}\right)\left(1+\frac{\bar{X}_{3}}{\bar{X}_{2}} \hat{\gamma}_{1}\right)}+\frac{\exp \left(\frac{-\hat{\gamma}_{1} \sigma^{2}}{\bar{X}_{3}}\right)}{\left(1+\frac{\bar{X}_{1}}{\bar{X}_{3}} \hat{\gamma}_{1}\right)\left(1+\frac{\bar{X}_{2}}{\bar{X}_{3}} \hat{\Gamma}\right)}\right) .
\end{aligned}
$$

When the case $n=4$ is examined, $4 !=24$ terms contribute to $P_{\text {out }}^{(1)}$; yet, it is sufficient to compute the term that corresponds to the $S_{1}=\{1,2,3,4\}$ set, that is expressed by

$$
I_{S_{1}}=\iiint \int_{\mathcal{D}_{1}} f_{1}\left(x_{(1)}\right) f_{2}\left(x_{(2)}\right) f_{3}\left(x_{(3)}\right) f_{4}\left(x_{(4)}\right) d x_{(4)} d x_{(3)} d x_{(2)} d x_{(1)}
$$


$\mathcal{D}_{1}$ now being given by

$$
\mathcal{D}_{1}=\left\{\begin{array}{l}
X_{(1)} \geq \hat{\gamma_{1}} \cdot\left(X_{(2)}+X_{(3)}+X_{(4)}+\sigma^{2}\right) \\
X_{(1)} \geq X_{(2)} \geq X_{(3)} \geq X_{(4)} \geq 0
\end{array} .\right.
$$

Here too, the assumption of exponential pdf allows to solve (56) in closed-form, resulting in

$$
\begin{gathered}
I_{S_{1}}=\exp \left(\frac{-\hat{\gamma}_{1} \sigma^{2}}{\bar{X}_{1}}\right) \\
\times \frac{\bar{X}_{1}^{3} \bar{X}_{2}^{2} \bar{X}_{3}}{\left(\bar{X}_{1}+\bar{X}_{2} \hat{\gamma}_{1}\right)\left(\bar{X}_{1} \bar{X}_{2}+\bar{X}_{1} \bar{X}_{3}+2 \bar{X}_{2} \bar{X}_{3} \hat{\gamma}_{1}\right)\left(\bar{X}_{1} \bar{X}_{2} \bar{X}_{3}+\bar{X}_{1} \bar{X}_{2} \bar{X}_{4}+3 \bar{X}_{2} \bar{X}_{3} \bar{X}_{4} \hat{\gamma}_{1}\right)} .
\end{gathered}
$$

At first sight, last expression might look unmanageable and hinder $P_{\text {out }}^{(1)}$ determination. Yet, in analogy with the previous $n=3$ case, the contribution in (57) has to be grouped with other conveniently identified terms, namely, those that correspond to the sets $S_{2}=\{1,2,4,3\}$, $S_{3}=\{1,3,2,4\}, S_{4}=\{1,3,4,2\}, S_{5}=\{1,4,2,3\}$ and $S_{6}=\{1,4,3,2\}$, leading to the partial sum

$$
C_{1}=\sum_{i=1}^{6} S_{i}=\frac{\exp \left(-\frac{\hat{\gamma}_{1} \sigma^{2}}{\bar{X}_{1}}\right)}{\left(1+\frac{\bar{X}_{2}}{\bar{X}_{1}} \hat{\gamma}_{1}\right)\left(1+\frac{\bar{X}_{3}}{\bar{X}_{1}} \hat{\gamma}_{1}\right)\left(1+\frac{\bar{X}_{4}}{\bar{X}_{1}} \hat{\gamma}_{1}\right)}
$$

In an analogous manner, 3 more partial sums are computed, so that altogether 4 terms are identified, $C_{k}, k=1,2,3,4$, the generic $C_{k}$ being

$$
C_{k}=\frac{\exp \left(-\frac{\hat{\gamma}_{1} \sigma^{2}}{\bar{X}_{k}}\right)}{\prod_{\substack{i=1 \\ i \neq k}}^{4}\left(1+\frac{\bar{X}_{i}}{\bar{X}_{k}} \hat{\Gamma}\right)}
$$

and $P_{\text {out }}^{(1)}$ is then computed as

$$
P_{\text {out }}^{(1)}=1-\sum_{k=1}^{4} C_{k}
$$

For an arbitrary number $n$ of superimposed signals, $n$ partial sums, each with $(n-1)$ ! elements, have to be determined. By induction, the generic sum $C_{k}$ turns out to be

$$
C_{k}=\frac{\exp \left(-\frac{\hat{\gamma}_{1} \sigma^{2}}{\bar{X}_{k}}\right)}{\prod_{\substack{i=1 \\ i \neq k}}^{n}\left(1+\frac{\bar{X}_{i}}{\bar{X}_{k}} \cdot \hat{\gamma}_{1}\right)}
$$

so that $P_{\text {out }}^{(1)}$, the probability that power-domain NOMA cannot guarantee the target data rate to the strongest user, is finally written as 


$$
P_{\text {out }}^{(1)}=1-\sum_{k=1}^{n} C_{k}=1-\sum_{k=1}^{n} \frac{\exp \left(\frac{-\hat{\gamma}_{1} \sigma^{2}}{\bar{X}_{k}}\right)}{\prod_{\substack{i=1 \\ i \neq k}}^{n}\left(1+\frac{\bar{X}_{i}}{\bar{X}_{k}} \hat{\gamma}_{1}\right)} .
$$

under the condition $\hat{\gamma}_{1} \geq 1 \mathrm{bits} / \mathrm{s} / \mathrm{Hz}$.

\section{REFERENCES}

[1] N. Varsier, L.-A. Dufrène, M. Dumay, Q. Lampin and J. Schwoerer, "A 5G New Radio for Balanced and Mixed IoT Use Cases: Challenges and Key Enablers in FR1 Band," in IEEE Communications Magazine, vol. 59, no. 4, pp. 82-87, April 2021.

[2] K. Senel, H. V. Cheng, E. Björnson and E. G. Larsson, "What Role can NOMA Play in Massive MIMO?," in IEEE Journal of Selected Topics in Signal Processing, vol. 13, no. 3, pp. 597-611, June 2019.

[3] L. Dai, B. Wang, Y. Yuan, S. Han, C. I and Z. Wang, "Non-orthogonal Multiple Access for 5G: Solutions, Challenges, Opportunities, and Future Research Trends," in IEEE Communications Magazine, vol. 53, no. 9, pp. 74-81, September 2015.

[4] S. M. Riazul Islam, N. Avazov, O. A. Dobre and K.-S. Kwak, "Power-domain Non-Orthogonal Multiple Access (NOMA) in 5G Systems: Potentials and Challenges," in IEEE Communications Surveys \& Tutorials, vol. 19, no. 2, pp. 721-742, October 2016.

[5] Z. Ding, X. Lei, G. K. Karagiannidis, R. Schober, J. Yuan, and V. J. Bhargava, "A Survey on Non-Orthogonal Multiple Access for 5G networks: Research Challenges and Future Trends," in IEEE Journal on Selectes Areas in Communications, vol. 35, no. 10, pp. 2181-2195, October 2017.

[6] B. Makki, K. Chitti, A. Behravan and M. Alouini, "A Survey of NOMA: Current Status and Open Research Challenges," in IEEE Open Journal of the Communications Society, vol. 1, pp. 179-189, January 2020.

[7] H. Tabassum, E. Hossain and J. Hossain, "Modeling and Analysis of Uplink Non-Orthogonal Multiple Access in LargeScale Cellular Networks Using Poisson Cluster Processes," in IEEE Transactions on Communications, vol. 65, no. 8, pp. 3555-3570, Aug. 2017.

[8] M. Al-Imari, P. Xiao, M. A. Imran, R. Tafazolli, ”Uplink non-orthogonal multiple access for 5G wireless networks,” 2014 11th International Symposium on Wireless Communications Systems (ISWCS), Barcelona, Spain, 2014.

[9] N. Zhang, J. Wang, G. Kang and Y. Liu, "Uplink Nonorthogonal Multiple Access in 5G Systems," in IEEE Communications Letters, vol. 20, no. 3, pp. 458-461, March 2016.

[10] Y. Liu, M. Derakhshani and S. Lambotharan, "Outage Analysis and Power Allocation in Uplink Non-Orthogonal Multiple Access Systems," in IEEE Communications Letters, vol. 22, no. 2, pp. 336-339, February 2018.

[11] Y. Gao, B. Xia, K. Xiao, Z. Chen, X. Li and S. Zhang, "Theoretical Analysis of the Dynamic Decode Ordering SIC Receiver for Uplink NOMA Systems," in IEEE Communications Letters, vol. 21, no. 10, pp. 2246-2249, October 2017.

[12] Y. Gao, B. Xia, K. Xiao, Z. Chen, X. Li and S. Zhang, "Analysis of the Dynamic Ordered Decoding for Uplink NOMA systems with imperfect CSI," in IEEE Transactions on Vehicular Technology, vol. 67, no. 7, pp. 6647-6651, July 2018.

[13] A. Agarwal, R. Chaurasiya, S. Rai and A. K. Jagannatham, ”Outage Probability Analysis for NOMA Downlink and Uplink Communication Systems With Generalized Fading Channels," in IEEE Access, vol.8, pp. 220461-220481, December 2020.

[14] A. Alqahtani, E. Alsusa, A. Al-Dweik and M. Al-Jarrah, "Performance Analysis for Downlink NOMA Over $\alpha$ - $\mu$ Generalized Fading Channels," in IEEE Transactions on Vehicular Technology, vol. 70, no. 7, pp. 6814-6825, July 2021. 
[15] R.J. Vaughan and W.N. Venables, "Permanent Expressions for Order Statistics Densities," in Journal of the Royal Statistical Society, vol. 34, no. 2, pp. 308-310, 1972.

[16] M.G. Kendall and A. Stuart, "The Advanced Theory of Statistics," 2nd edition, vol. 1, 1958.

[17] J. M. Holtzman, "A simple, accurate method to calculate spread-spectrum multiple-access error probabilities," in IEEE Transactions on Communications, vol. 40, no. 3, pp. 461-464, March 1992. 